\title{
Swelling Properties of Chitosan/CMC/PEGDA based Semi-IPN Hydrogel
}

\author{
Jong Shin Park, Ji Hyun Yeo \\ Seoul National University \\ 1 Gwanak-ro, Gwanak-gu, Seoul 08826, South Korea \\ jongshin@snu.ac.kr; JiHyunYeo@snu.ac.kr
}

\section{Extended Abstract}

Hydrogels are three-dimensional and hydrophilic polymer which can be formed by cross-linking polymer chain and can swell but not dissolve in water. It can be applied to many fields, such as agriculture, hygiene products, wastewater treatment, and drug delivery system. Semi-interpenetrating polymer networks (semi-IPN) is a way of blending two polymers where only one is crosslinked in the presence of another to produce and additional non-covalent interaction between the two polymers.

Traditionally, most of the materials used to make hydrogels are synthetic polymers [1]. However, petroleum-based synthetic polymers have some disadvantages which are toxicity and poor environmental friendly properties. So, natural polymer can overcome the synthetic polymer's disadvantages.

Chitosan is derived from chitin and second most abundant natural polymer. It is soluble only in acidic conditions. It has abundant $-\mathrm{NH} 2$, which can be used to form many kinds of bond with other functional groups. Chitosan is an cationic polymer [2].[3]. Carboxymethyl cellulose(CMC) is an anionic water-soluble natural polymer derivative. So, by solution blending of two natural polyelectrolyte, CS and CMC can form a natural amphoteric hydrogel [4]. In addition, a biocompatible crosslinker, PEGDA can react with chitosan via Michael reaction, so they can be form a semi-IPN polymer.

Completed hydrogels can absorb a lot of water, so they can be applied to biocompatible superabsorbents(SAPs), drug delivery system(DDS) and anatural polyelectrolyte hydrogels.

\section{References}

[1] W. A. Laftah, S. Hashim \& A. N. Ibrahim, "Polymer Hydrogels: A Review," Polymer-Plastics Technology and Engineering, vol. 50, pp. 1475-1486, 2011.

[2] R. Czechowska-Biskup, R. A. Wach, P. Stojek, M. Kamińska, J. M. Rosiak, P. Ulański, "synthesis of chitosan and carboxymethyl chitosan hydrogels by electron beam irradiation," Progress on Chemistry and Application of Chitin and its Derivatives, vol.12, pp. 27-45, 2016

[3] B.-L. Guo and Q.-Y. Gao, "Preparation and properties of a pH/temperature-responsive carboxymethyl chitosan/poly(N-isopropylacrylamide)semi-IPN hydrogel for oral delivery of drugs," Carbohydrate Research, vol. 342, pp. 2416-2422, 2007.

[4] J. Shang, Z. Shao and X. Chen, "Electrical Behavior of a Natural Polyelectrolyte Hydrogel: Chitosan/Carboxymethylcellulose Hydrogel," Biomacromolecules, vol. 9, pp. 1208-1213, 2008. 